\title{
Correction to: A literature review and novel theoretical approach on the optical properties of whole blood
}

\author{
Nienke Bosschaart ${ }^{1} \cdot$ Gerda J. Edelman $^{1} \cdot$ Maurice C. G. Aalders ${ }^{1} \cdot$ Ton G. van Leeuwen $^{1} \cdot$ Dirk J. Faber $^{1}$
}

Published online: 25 October 2021

○) Springer-Verlag London Ltd., part of Springer Nature 2021

Correction to: Lasers Med Sci

https://doi.org/10.1007/s10103-013-1446-7

Original text, taken from the on-line version:

Following the method of Duysens, adapting only the terminology, we arrive at:

$\mu_{a, b l o o d}=\left(\frac{1-e^{\left(\mu_{a, H b} \bullet d_{\mathrm{RBC}}\right)}}{\mu_{a, H b} \bullet d_{\mathrm{RBC}}}\right) \mu_{a}, H B$

where $\mu_{\mathrm{a}, \mathrm{blood}}$ and $\mu_{\mathrm{a}, \mathrm{Hb}}$ are the absorption coefficient of a blood sample and haemoglobin solution, respectively. The length $d_{\mathrm{RBC}}$ is a typical dimension of a red blood cell.

There are two issues:

- There is a minus-sign missing within the exponential.

- The subscripts of the absorption coefficients within the parenthesis (..) are incorrect, and accordingly, the first sentence after the Eq. is incomplete.

The correct version should read:

Following the method of Duysens [32], adapting only the terminology, we arrive at:

The original article can be found online at https://doi.org/10.1007/ s10103-013-1446-7.

Nienke Bosschaart

n.bosschaart@amc.uva.nl

1 Biomedical Engineering and Physics, Academic Medical Center, University of Amsterdam, P.O. Box 22700,

1100 DE Amsterdam, The Netherlands $\mu_{a, b l o o d}=\left(\frac{1-e^{-\mu_{a, R B C} \bullet d_{R B C}}}{\mu_{a, R B C} \bullet d_{R B C}}\right) \mu_{a, H b}$

where $\mu_{a, b l o o d}$ and $\mu_{a, H b}$ are the absorption coefficient of a blood sample and haemoglobin solution, respectively, and $\mu_{a, R B C}$ is the absorption coefficient of the hemoglobin solution inside the red blood cell. The last two absorption coefficients are related through the haematocrit, $\mu_{a, H b}=H c t \cdot \mu_{a, R B C}$. The length $d_{\mathrm{RBC}}$ is a typical dimension of a red blood cell.

The original article has been corrected.

Publisher's note Springer Nature remains neutral with regard to jurisdictional claims in published maps and institutional affiliations. 